Canadian Oncology

Nursing Journal

Revue canadienne

de soins infirmiers

en oncologie

Volume 28, Issue 4 • Fall 2018

elSSN: 2368-8076 


\title{
Programme d'infirmière-coordonnatrice des soins du cancer du sein : effet sur le niveau de détresse des patientes
}

\author{
par Savitri Singh-Carlson, Frances Wong, Kris Trevillion et Grace Reynolds
}

\section{RÉSUMÉ}

La présente étude visait à déterminer si les niveaux d'anxiété et de dépression des patientes bénéficiant du soutien et de la navigation d'une coordonnatrice des soins $d u$ cancer $d u$ sein (CSCS) avant leur première consultation en oncologie au centre Abbotsford de la British Columbia Cancer Agency (BCCA) différaient de ceux des patientes du centre n'ayant pas reçu ce même soutien. Une étude rétrospective a comparé les scores de dépistage psychologique pour le cancer (Psychological Screen for Cancer - PSSCAN) d'un groupe témoin constitué de patientes qui avaient reçu un diagnostic de cancer du sein récemment et qui recevaient les soins oncologiques habituels aux scores de patientes accompagnées par une CSCS avant leur consultation en oncologie (groupe à l'étude). Au total, 91 scores ont été analysés : 54 dans le groupe à l'étude et 37 dans le groupe témoin. Aucune différence significative n'a été décelée entre les scores PSSCAN d'anxiété et de dépression des deux groupes.

\section{INTRODUCTION}

T es femmes qui reçoivent un diagnostic de cancer du sein ـ pour la toute première fois sont confrontées à toute une gamme d'émotions, quels que soient leur âge ou leur origine ethnique (Hewitt, Greenfield et Stovall, 2005; Pederson, Sawatzky et Hack, 2010). On pourrait donc supposer que l'instigation d'un programme de dépistage de routine de la détresse psychosociale contribuerait à calmer l'anxiété des femmes pendant le processus diagnostique (Carlson, Groff, Maciejewski et Bultz, 2010). L'American College of Surgeons et sa Commission on Cancer ont d'ailleurs adopté une norme obligeant les programmes de traitement du cancer à se doter d'un outil de dépistage de la détresse psychologique pour

\section{AU SUJET DES AUTEURES}

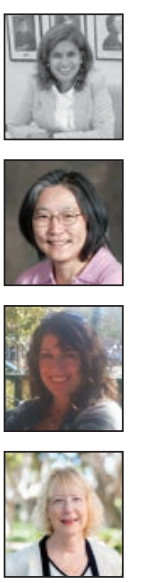

Savitri Singh-Carlson, Ph.D., APHN-BC, FAAN, Professeure, San Diego State University, École de sciences infirmières, San Diego, CA, USA, 92182. Courriel : ssinghcarlson@sdsu.edu

Frances Wong, RN, Breast Cancer Co-ordinator, BCCA, FVC

Kris Trevillion, MD, FRCPC(C), BCCA, FVC

Grace Reynolds, Professor, California State University, Long Beach, Health Care Administration, Graduate Program Director, Director, Center for Behavioral Research and Services

DOI: $10.5737 / 23688076284256261$ évaluer l'état émotionnel des patientes au moment du diagnostic et tout au long du traitement (Buxton, Lazenby, Daugherty et al., 2014). La plupart des femmes atteintes de cancer du sein vivront, entre autres émotions, une intensification de l'anxiété et de la dépression les empêchant de composer avec le diagnostic et le traitement (Linden, Yi, Barroetavena, MacKenzie et Doll, 2005; Campbell, Craig, Eggert et Bailey-Dorton, 2010; Pederson, Sawatsky et Hack, 2010; Case, 2011; Pederson et Hack, 2011). Les données montrent que les infirmières pivots spécialisées peuvent alléger le stress et l'anxiété des patientes en leur offrant du soutien psychosocial, ainsi que la bonne information au bon moment, tout en veillant à bien structurer la continuité des soins (Campbell, Craig, Eggert et BaileyDorton, 2010; Case, 2011; Liebert, Parle, White et Rodger, 2011; Lim, Devi et Ang, 2011; Pederson et Hack, 2011; Fillion, Cook, Veillette, Aubin, DeSerres et al., 2012). Ces données sont confirmées par la National Coalition of Oncology Nurse Navigators et appuyées par d'autres études aux résultats similaires (Winstead, 2012; National Cancer Institute, 2017).

Les patientes ayant reçu un premier diagnostic de cancer et les traitements subséquents ont mentionné l'importance d'une approche multidisciplinaire, mais elles ont toutefois souffert du manque de structure au niveau de la coordination des différents aspects des soins dans le système de santé (Hryniuk, Simpson, McGowan et Carter, 2014; Singh-Carlson, Gotz, 2014). À cause de ces lacunes de structure et de coordination, les femmes se sentent généralement mal accompagnées aux heures les plus difficiles de leur expérience du cancer du sein (Drageset, Lindstrom et Underlid, 2009).

Le centre Abbotsford de la British Columbia Cancer Agency (BCCA) a reconnu le besoin d'attribuer à une infirmière en oncologie un rôle de soutien spécialisé auprès des patientes atteintes de cancer du sein avant leur aiguillage vers un centre de traitement. Il a donc créé un programme ciblé de soins de soutien, ainsi que le poste de coordonnatrice des soins du cancer du sein (CSCS). Les infirmières qui revêtent le rôle de coordonnatrice possèdent de l'expérience en oncologie et travaillent à la fois au centre de traitement du cancer et à la clinique de santé du sein de l'hôpital affilié (hôpital régional Abbotsford). La mission de la CSCS consiste à servir de lien, de guide et de source informationnelle pour soutenir la patiente. Selon Hryniuk, Simpson, McGowan et Carter (2014), les soins de soutien n'ont fait leur apparition que récemment dans les recherches sur le traitement contre le cancer. Ils reposent sur le principe qu'il est habituellement bénéfique pour les patientes de recevoir de l'information détaillée sur la santé, de connaître le déroulement du protocole de traitement 
et d'être actives dans leurs propres soins - le tout dans le cadre d'une démarche multidisciplinaire à laquelle participent les professionnels de la santé.

\section{But de l'étude}

Le but de la présente étude était de déterminer si les patientes jouissant du soutien et de la navigation d'une CSCS avant leur première consultation en oncologie au centre Abbotsford de la BCCA présentaient des niveaux d'anxiété et de dépression différents de ceux des patientes qui avaient reçu les soins habituels sans profiter de ce soutien supplémentaire. Pour ce faire, les auteures ont procédé à un examen rétrospectif des dossiers des patientes afin de comparer leurs réactions au soutien social et l'influence de cette variable sur les items d'anxiété et de dépression du questionnaire sur le dépistage psychologique pour le cancer (Psychological Screen for Cancer PSSCAN). Le questionnaire PSSCAN est un outil de dépistage spécialement conçu pour déceler, en contexte clinique, l'anxiété et la dépression chez les patients atteints de cancer (Linden, Yi, Barroetavena, MacKenzie et Doll, 2005). Il est couramment utilisé dans les milieux cliniques de la BCCA pour dépister la détresse. Notons que l'instrument a été revalidé aux fins de la présente étude (Linden, Vodermaier, MacKenzie et al., 2009).

\section{LA CLINIQUE DE SANTÉ DU SEIN ET LE RÔLE DE COORDONNATRICE DES SOINS DU CANCER}

La clinique de santé du sein est le fruit d'une collaboration entre l'hôpital régional Abbotsford et le centre Abbotsford de la BCCA. Unique en son genre, cette collaboration est née en juin 2011, lorsqu'on a constaté un manque du côté des services essentiels, de la coordination des communications et de la continuité des soins pour les patientes dont les résultats mammographiques étaient anormaux (Trevillion, Singh-Carlson, Wong et Sherriff, 2015). Cet exceptionnel partenariat de santé collaborative a mené à la création d'un programme spécialisé offrant des soins ciblés aux femmes chez qui on redoute un cancer du sein, du dépistage au diagnostic. L'objectif de ce programme novateur de la BCCA est de soutenir les patientes atteintes de cancer du sein dès le début de la trajectoire des soins. Habituellement, quand la patiente passe une mammographie dans le cadre du programme de dépistage de la BCCA, le processus diagnostique s'enclenche dès la détection d'une anomalie mammographique. La patiente est ensuite aiguillée vers une clinique de santé du sein et reçoit un rendez-vous pour d'autres examens et une biopsie. Elle voit aussi un généraliste ou un chirurgien, qui procède à un examen clinique et à une mammographie avec ou sans échographie, le cas échéant. Lorsque la biopsie vient confirmer le cancer, c'est le chirurgien de la clinique de santé du sein qui présente le bilan des analyses à la patiente peu de temps après l'obtention des résultats. Règle générale, la coordonnatrice rencontre la patiente le jour même, mais elle peut aussi lui téléphoner dans la journée ou le jour suivant pour répondre à ses questions. La patiente sera ensuite aiguillée vers le centre de traitement pour une consultation en oncologie. Le processus, de l'annonce du diagnostic à la première consultation en oncologie, prend de 7 à 10 semaines en moyenne.
La coordonnatrice des soins du cancer du sein (CSCS) est un chaînon important de l'équipe interdisciplinaire; elle connaît les normes, les protocoles, les politiques et les lignes directrices en matière de pratiques exemplaires de la BCCA, de même que la trajectoire des soins que devra suivre la patiente, la physiopathologie du cancer du sein, les procédures diagnostiques, la chirurgie d'ablation de la tumeur et de reconstruction du sein, ainsi que les traitements systémiques et la radiothérapie. La patiente qui vient de recevoir un diagnostic de cancer repart avec une trousse d'information contenant l'ouvrage Intelligent Patient Guide to Breast Cancer (BC Cancer Agency, 2017), ainsi que des brochures sur la nutrition, les exercices post-chirurgicaux, le soutien familial et la survie au cancer. Grâce au processus initial d'évaluation infirmière, la CSCS aide aussi la patiente en répondant à ses besoins émotionnels et en lui fournissant des ressources à cet effet. Elle lui donne également de l'information sur le soutien communautaire disponible et la dirige vers le counseling à l'intention des patientes et de leurs familles qu'offre la BCCA. Cet aiguillage vers les services psychosociaux, que pratiquent tous les professionnels de la santé, est vu comme le meilleur facteur prédictif de la diminution de l'anxiété et de la dépression lorsque les conditions de dépistage et de triage sont optimales (Carlson, Groff, Maciejewski et Bultz, 2010).

Le service de counseling de la BCCA est une ressource de soutien social pour les patients et leurs familles. On le propose automatiquement aux patients dont le score PSSCAN indique la présence d'anxiété ou de dépression. Les patients peuvent y être dirigés par n'importe quel professionnel de la santé ou faire eux-mêmes une demande de consultation. Au besoin, la CSCS fait le pont entre la patiente, les ressources communautaires et les services de soutien. Elle demeure également disponible pour rencontrer la patiente et sa famille en personne, au téléphone ou par Internet. La CSCS peut aussi aiguiller vers le centre de la BCCA pour des consultations en oncologie, le cas échéant. Le fait que la CSCS doive jouer le rôle d'infirmière pivot a été considéré comme une lacune des soins. Il fallait créer un nouveau rôle où l'infirmière s'occuperait d'apporter information et du soutien aux femmes dès la première consultation en oncologie (Trussler, 2001).

Dans les pratiques actuelles et habituelles de la BCCA, tous les nouveaux patients qui se rendent à leur premier rendez-vous remplissent le questionnaire sur le dépistage psychologique pour le cancer (Psychological Screen for Cancer - PSSCAN) (Linden, Yi, Barroetavena, MacKenzie et Doll, 2005; Linden, Vodermaier, MacKenzie et al., 2009). Ce questionnaire évalue, en 21 items, le soutien social perçu, le soutien social désiré, la qualité de vie liée à la santé, l'anxiété et la dépression. En cas d'anxiété et de dépression (qui peuvent être perçues comme de la détresse), il est possible d'appliquer les interventions psychosociales dès la première rencontre afin d'améliorer la qualité de vie des patients et les aider, ainsi que leur famille, à mieux gérer un possible diagnostic de cancer (Buxton, Lazenby, Daugherty et al., 2014). Malheureusement, la détresse passe souvent sous le radar et n'est alors pas traitée (Carlson, Groff, Maciejweski et Bultz, 2010). Le patient doit remplir le questionnaire de dépistage avant la consultation en 
oncologie, habituellement le même jour. À partir des résultats, les professionnels de la santé peuvent détecter les patients aux prises avec de l'anxiété et des symptômes de dépression plus intenses. Ces patients et leur famille sont ensuite dirigés vers les services de counseling de la BCCA.

\section{MÉTHODOLOGIE}

L'étude compare rétrospectivement les scores PSSCAN de patientes en oncologie ayant reçu un diagnostic de cancer du sein entre janvier 2010 et décembre 2012. Dans le groupe témoin, les patientes n'avaient pas reçu les soins de la CSCS avant leur visite de consultation. Dans le groupe à l'étude, les femmes avaient bénéficié de l'aide de la CSCS avant leur consultation en oncologie. La majorité des patientes avaient reçu un résultat anormal à la mammographie de dépistage et avaient alors été aiguillées par la CSCS vers la clinique de santé du sein pour subir d'autres examens afin de possiblement confirmer le diagnostic. C'est à ces patientes que se restreignent les deux groupes de la présente étude.

Pour le groupe témoin, les critères d'inclusion étaient les suivants : a) mammographie de dépistage anormale; b) diagnostic de cancer du sein confirmé par biopsie; c) questionnaire PSSCAN rempli lors de la consultation initiale en oncologie à la BCCA. Pour le groupe à l'étude, ces critères étaient : a) mammographie de dépistage anormale; b) diagnostic de cancer du sein confirmé par biopsie; c) soins de la CSCS reçus avant la consultation en oncologie à la BCCA; d) questionnaire PSSCAN rempli lors de la consultation initiale en oncologie à la BCCA. Et pour les deux groupes, voici les critères d'exclusion retenus : a) ne pas avoir rempli le questionnaire PSSCAN en entier; b) souffrir d'un cancer du sein avancé avec métastases; c) avoir déjà eu un cancer du sein.

Questionnaire PSSCAN : En raison de ses propriétés psychométriques, le questionnaire PSSCAN est un outil de dépistage d'une utilité suffisante et peut même faciliter le suivi du bien-être émotionnel des patients; c'est, du moins, ce qu'indique la recherche (Linden, Yi, Barroetavena, MacKenzie et Doll, 2005; Linden, Vodermaier, MacKenzie et al., 2009). Expressément conçu pour les patients atteints de cancer, le questionnaire PSSCAN se divise en trois sections (A, B et C) évaluant 21 items. À la BCCA, il est intégré aux pratiques cliniques de routine depuis un certain temps déjà. Néanmoins, en avril 2012, la BCCA a révisé la version 2005 du PSSCAN et c'est maintenant ce questionnaire révisé, le PSSCAN-R, qui est utilisé. Dans les deux versions (PSSCAN et PSSSCAN-R), la partie $\mathrm{A}$ est identique, et les questions 1 à 5 demeurent les mêmes; la question 6 a toutefois été supprimée. Dans la partie $\mathrm{B}$, le format des questions a été totalement modifié. Rien n'a été changé dans la partie C. Comme les deux versions diffèrent quelque peu, nous avons décidé de n'inclure à l'étude que les réponses aux questions 1 à 5 de la partie $\mathrm{A}$, de même que toutes les questions de la partie C du PSSCAN, afin d'obtenir des données similaires à celles de la version révisée PSSCANR. Il s'agit là d'un point important, puisque nous comparons l'efficacité des réponses fournies au PSSCAN. Les sous-échelles du PSSCAN, dans la partie C par exemple, permettent de mesurer efficacement l'anxiété et la dépression des patients atteints de cancer du sein et du poumon (Carlson, Groff, Maciejewski et Bultz, 2010). Malheureusement, comme aucune autre étude n'utilisait le questionnaire PSSCANR, nous n'avons pas pu valider la pertinence de la version révisée.

L'utilité du questionnaire PSSCAN ayant été établie, l'hypothèse nulle est la suivante : les nouvelles patientes qui remplissent le questionnaire PSSCAN et qui reçoivent en plus des soins de soutien de la CSCS avant leur consultation en oncologie présentent des niveaux d'anxiété et de dépression semblables à ceux des patientes ayant seulement rempli le questionnaire PSSCAN.

Outre les scores PSSCAN de chaque patiente, certaines données démographiques ont également été recueillies, car elles pouvaient influencer les scores. Il s'agissait de : l'âge au moment du diagnostic; les conditions de logement, l'état civil, le soutien social, les préoccupations financières, les personnes à charge, la barrière linguistique (besoin d'un interprète) et les antécédents psychologiques.

\section{Analyse des données}

Aux fins de l'analyse, une valeur numérique a été assignée à chacune des questions. Les différences entre les deux groupes de participantes ont été mesurées à l'aide de statistiques descriptives. L'analyse a ainsi permis de comparer les réponses des deux groupes afin de repérer les différences subjectives quant à l'expérience vécue par les patientes à leur première visite à la clinique de santé du sein de la BCCA. Toutes les analyses ont été réalisées avec la version 9.3 de SAS (Hatcher, 2003).

\section{RÉSULTATS}

$\mathrm{Au}$ total, la présente étude a examiné les questionnaires PSSCAN de 9I patientes, dont 54 dans le groupe à l'étude et 37 dans le groupe témoin. Les données sur la démographie et le soutien social sont présentées au tableau 1. Il y avait notamment davantage de femmes vivant seules dans le groupe témoin. Quant à celles du groupe à l'étude, elles étaient plus susceptibles d'avoir des personnes à charge dépendant de leurs soins et de leur aide. Ce sont les deux seules variables démographiques à différer significativement entre les deux groupes. Il n'y avait aucune différence pour ce qui est des difficultés linguistiques $\left(\mathrm{c}^{2}(1)=1,58, \mathrm{p}=.20\right)$, des antécédents psychiatriques $\left(\mathrm{c}^{2}(1)=0,0057, \mathrm{p}=.93\right)$, et des préoccupations financières passées $\left(c^{2}(1)=1,45, p=.22\right)$.

Le tableau 2 détaille les scores moyens obtenus au questionnaire PSSCAN pour les items touchant l'anxiété et la dépression. Dans un cas seulement, le score moyen était significativement plus élevé pour l'un des groupes (en l'occurrence le groupe à l'étude); il s'agissait de l'affirmation suivante : « Au cours de la dernière semaine, j'ai perdu de l'intérêt pour des choses que j'aime ou que j'apprécie d'habitude. »

À partir des données des deux sous-échelles du PSSCAN pour lesquelles les données, le soutien social, l'anxiété et la dépression étaient similaires, on a déterminé, à l'aide de tests-t pour échantillons indépendants, la différence existant entre les scores moyens du groupe témoin et du groupe à l'étude. Sur la sous-échelle du soutien social, les femmes du groupe témoin 
Tableau 1 : Comparaison du groupe à l'étude et du groupe témoin (données démographiques et soutien social)

\begin{tabular}{|l|c|c|c|}
\hline Variable & $\begin{array}{c}\text { Groupe } \\
\text { à l'étude } \\
\text { (N=54) }\end{array}$ & $\begin{array}{c}\text { Groupe } \\
\text { témoin } \\
\text { (N=37) }\end{array}$ & $\mathbf{c}^{2}$ \\
\hline & $\mathbf{N}(\%)$ & $\mathbf{N}(\%)$ & \\
\hline Âge & $\begin{array}{c}\text { Moyenne } \\
\text { (ÉT) }\end{array}$ & $\begin{array}{c}\text { Moyenne } \\
\text { (ÉT) }\end{array}$ & $\mathbf{t}$ \\
\hline $\begin{array}{l}\text { Temps entre les } \\
\text { évaluations/valeur de } \\
\text { référence }\end{array}$ & $\begin{array}{c}82,05(10,1) \\
(28,89)\end{array}$ & $65,9(8,42)$ & 1,93 \\
\hline
\end{tabular}

Avez-vous eu besoin d'un interprète?

\begin{tabular}{|l|c|c|c|}
\hline Oui & $5(100)$ & $0(0)$ & \\
\hline Non & $48(56)$ & $37(44)$ & $3,69^{*}$ \\
\hline
\end{tabular}

Avez-vous des personnes à charge qui dépendent de vos soins?

\begin{tabular}{|l|c|c|c|}
\hline Oui & $12(86)$ & $2(14)$ & \\
\hline Non & $41(55)$ & $34(45)$ & $4,72^{* *}$ \\
\hline
\end{tabular}

Vivez-vous seule?

\begin{tabular}{|l|l|l|l|}
\hline Oui & $6(33)$ & $12(67)$ & \\
\hline Non & $47(65)$ & $25(35)$ & $6,07^{* * *}$ \\
\hline
\end{tabular}

Lorsque vous avez besoin d'aide, pouvez-vous compter sur quelqu'un pour vous assister dans vos tâches quotidiennes, comme les courses, la cuisine, le transport?

\begin{tabular}{|c|c|c|c|}
\hline Oui & $44(56)$ & $35(44)$ & \\
\hline Non & $7(78)$ & $2(22)$ & 1,61 \\
\hline
\end{tabular}

Avez-vous des contacts réguliers avec des amis ou des membres de votre famille?

\begin{tabular}{|c|c|c|c|}
\hline Oui & $53(60)$ & $36(40)$ & \\
\hline Non & $1(50)$ & $1(50)$ & 0,07 \\
\hline
\end{tabular}

Avez-vous perdu votre conjoint au cours des dernières années?

\begin{tabular}{|l|l|l|l|}
\hline Oui & $4(44)$ & $5(55)$ & \\
\hline Non & $47(61)$ & $30(39)$ & 0,91 \\
\hline
\end{tabular}

Pouvez-vous compter sur quelqu'un pour vous apporter du soutien émotionnel?

\begin{tabular}{|l|c|c|c|}
\hline Oui & $50(58)$ & $36(42)$ & \\
\hline Non & $3(75)$ & $1(25)$ & 0,44 \\
\hline
\end{tabular}

Note. ${ }^{*} \mathrm{p}<.10 ;{ }^{* *} \mathrm{p}<.05 ;{ }^{* * *} \mathrm{p}<.01$.
Tableau 2 : Réponses moyennes aux questions sur les épisodes, passés et récents, d'anxiété et de dépression

\begin{tabular}{|c|c|c|c|}
\hline Variable & $\begin{array}{l}\text { Groupe } \\
\text { à l'étude } \\
(\mathrm{N}=54)\end{array}$ & $\begin{array}{l}\text { Groupe } \\
\text { témoin } \\
(\mathrm{N}=37)\end{array}$ & $c^{2}$ \\
\hline & $\mathbf{N}(\%)$ & $N(\%)$ & \\
\hline & Moyenne (ÉT) & Moyenne (ÉT) & $\mathbf{t}$ \\
\hline $\begin{array}{l}\text { Au cours de la dernière } \\
\text { semaine, j’ai ressenti } \\
\text { des palpitations et des } \\
\text { tremblements. }\end{array}$ & $1,17(0,69)$ & $1,16(0,55)$ & 0,03 \\
\hline $\begin{array}{l}\text { Au cours de la dernière } \\
\text { semaine, j’ai eu } \\
\text { l'impression de ne rien } \\
\text { contrôler. }\end{array}$ & $1,33(0,93)$ & $1,14(0,42)$ & 1,21 \\
\hline $\begin{array}{l}\text { Au cours de la dernière } \\
\text { semaine, j'ai perdu de } \\
\text { l'intérêt pour les choses } \\
\text { que j'aime d'habitude. }\end{array}$ & $1,29(0,71)$ & $1,03(0,37)$ & $2,10^{*}$ \\
\hline $\begin{array}{l}\text { Au cours de la dernière } \\
\text { semaine, je me suis sentie } \\
\text { nerveuse intérieurement. }\end{array}$ & $1,35(0,82)$ & $1,39(0,86)$ & 0,15 \\
\hline $\begin{array}{l}\text { Au cours de la dernière } \\
\text { semaine, j'étais tendue et } \\
\text { incapable de relaxer. }\end{array}$ & $1,41(0,90)$ & $1,37(0,75)$ & 0,16 \\
\hline $\begin{array}{l}\text { Au cours de la dernière } \\
\text { semaine, j’ai eu des } \\
\text { pensées répétitives et des } \\
\text { idées angoissantes. }\end{array}$ & $1,22(0,90)$ & $1,16(0,60)$ & 0,35 \\
\hline $\begin{array}{l}\text { Au cours de la dernière } \\
\text { semaine, j'étais agitée et } \\
\text { incapable de rester calme. }\end{array}$ & $1,29(0,81)$ & $1,21(0,47)$ & 0,54 \\
\hline $\begin{array}{l}\text { Dernièrement, j’ai eu des } \\
\text { idées suicidaires. }\end{array}$ & $0,92(0,38)$ & $0,95(0,32)$ & 0,26 \\
\hline $\begin{array}{l}\text { Au cours de la dernière } \\
\text { année, j’ai été triste } \\
\text { ou déprimée pendant } \\
2 \text { semaines ou plus. }\end{array}$ & $1,46(1,09)$ & $1,35(0,89)$ & 0,51 \\
\hline $\begin{array}{l}\text { Pendant } 2 \text { ans ou plus de } \\
\text { ma vie, j'ai été déprimée } \\
\text { ou triste, la plupart du } \\
\text { temps, même si parfois } \\
\text { j'allais bien. }\end{array}$ & $1,31(0,94)$ & $1,16(0,72)$ & 0,83 \\
\hline \multicolumn{4}{|l|}{ Note : ${ }^{*} p<.05$} \\
\hline
\end{tabular}

ont obtenu des scores significativement plus élevés $(\mathrm{M}=3,36$, ÉT $=0,76)$ comparativement au groupe à l'étude $(M=2,96$, ÉT $=0,60, t(59)=2,62, p=.01)$. Sur la sous-échelle de l'anxiété et de la dépression, il n'y avait aucune différence entre le groupe témoin $(\mathrm{M}=\mathrm{II}, 91, \mathrm{ET}=3,70)$ et le groupe à l'étude $(\mathrm{M}=12.77, \mathrm{ET}=5,89, \mathrm{t}(88)=\mathrm{ns})$. 


\section{DISCUSSION}

La présente étude explorait les répercussions sur la détresse d'un programme de coordonnatrice des soins du cancer du sein (CSCS) qui offrait du soutien aux femmes traversant une période d'attente critique entre la détection d'une tumeur suspecte et la pose du diagnostic. Cet unique partenariat de santé, de même que la collaboration entre le milieu hospitalier et le centre de la BCCA, ont été l'occasion d'apporter soutien et information aux femmes chez qui ont suspectait un cancer du sein, et ce, jusqu'au diagnostic. Les CSCS du programme, également infirmières en oncologie, pouvaient assurer la continuité des soins et orienter les femmes dans le système de santé dès le début de leur parcours.

La CSCS est un chaînon important de l'équipe interdisciplinaire et sert de pont entre la patiente et les ressources communautaires. En outre, la CSCS peut aiguiller, au besoin, les patientes et leur famille vers le counseling, une ressource de soutien social offerte par la BCCA. Lélaboration d'une démarche clinique axée sur la continuité des soins infirmiers pendant l'expérience du cancer devrait réduire la détresse et favoriser l'établissement de relations de confiance. Cette stratégie de soins continus aide également les patientes à mieux accepter le diagnostic de cancer, à mieux retenir l'information relative au traitement et, peut-être aussi, à mieux comprendre les soins de suivi post-traitement (Zabora, Brintzenhofeszoc, Curbow, Hooker et Piantadosi, 2001). Ces éléments devront éventuellement être évalués à l'aide de variables pertinentes et l'influence de la CSCS devra faire l'objet d'une étude plus poussée.

Les résultats de la présente étude ne montrent aucune différence significative entre le groupe témoin et le groupe à l'étude au moment de la consultation initiale en oncologie après un diagnostic positif. Toutefois, cette situation s'explique peut-être par le moment choisi pour la collecte des données. En effet, les femmes ont rempli le questionnaire PSSCAN au moment où elles sont sans doute le plus anxieuses, c'est-à-dire avant leur première consultation avec l'oncologue, où elles en sauront plus sur le diagnostic et les possibilités de traitement. La prochaine fois, il pourrait être préférable de recueillir les données à une autre occasion.

Après une revue systématique de la recherche à ce sujet, il appert que la navigation et le rôle de la CSCS influencent positivement plusieurs aspects de la trajectoire des soins des patientes atteintes de cancer du sein (Case, 2011; RobinsonWhite, Conroy, Slavish et Rozenzweig, 2010). À leur entrée dans la structure organisationnelle de traitement du cancer, les femmes reçoivent une quantité incroyable d'information; l'infirmière pivot semble jouer un rôle central pour apaiser le sentiment de peur et d'anxiété (Trevillion, Singh-Carlson, Wong et Sherriff, 2015). D'autres recherches seront nécessaires pour bien comprendre la mission de la CSCS et son influence sur les patientes pendant la période entourant l'établissement du diagnostic.

Dès le premier rendez-vous, les patientes ont besoin de soutien psychologique. Il faudrait toutefois mettre sous la loupe l'efficacité du questionnaire de dépistage PSSCANR lorsqu'il est utilisé pendant la visite initiale en oncologie et lors des consultations subséquentes afin de bien cerner les différences entre les niveaux d'anxiété. En administrant le questionnaire PSSCAN-R plusieurs fois (c.-à-d. au rendu du diagnostic ainsi qu'au cours des visites suivantes, au besoin), nous pourrions voir s'il surgit des différences entre les données de référence et les données du suivi. Les résultats de Carlson, Groff, Maciejewski et Bultz (2010) vont dans le même sens que la présente étude; les auteurs rapportent un niveau de détresse moins élevé au rendez-vous de suivi qu'au moment du dépistage initial du cancer du sein. D'autres rapports indiquent que le dépistage et la gestion de la détresse améliorent généralement l'état des patientes; lorsque les oncologues sont sensibilisés au dépistage de l'anxiété et de la dépression, ils peuvent encourager les patientes à discuter de leur situation (Buxton, Lzenby, Daugherty et al., 2014). En parlant ouvertement de leur détresse émotionnelle, les patientes pourront mieux gérer leur état psychosocial et seront plus satisfaites de leurs traitements, ce qui embellira leur qualité de vie. Encore une fois, ces aspects doivent être envisagés à la lumière de la détresse entourant la période du diagnostic.

La présente étude visait à établir si les patientes recevant de l'information et des soins de soutien et de navigation d'une CSCS avant leur première consultation en oncologie, dans un centre de la BCCA, manifestaient des niveaux d'anxiété et de dépression différents des patientes fréquentant un centre de la BCCA, sans recevoir ce même type de soutien. La National Coalition of Oncology Nurse Navigators (2017) se dit en faveur du rôle de navigatrice pour aider les patientes atteintes d'un cancer du sein à apaiser l'anxiété causée par une mammographie anormale. C'est en puisant dans ses aptitudes de communication interpersonnelle (à l'écrit, à l'oral ou par son écoute active) que l'infirmière pourra défendre les intérêts des patientes afin qu'elles reçoivent des soins centrés sur leurs besoins. Pendant la période entourant l'établissement du diagnostic et pour pouvoir apporter aux patientes de l'information et du soutien à la fois utiles et respectueux de leur culture, il faut évaluer efficacement et dès le début leur niveau de détresse, de même que leur capacité et leurs modes d'apprentissage.

\section{Limites}

La présente étude présente certaines limites, qu'il importe de mentionner. D'abord, l'échantillon était restreint; des travaux portant sur un groupe plus important de femmes suivies par une infirmière pivot pourraient révéler des différences que la présente étude n'a pas été en mesure de détecter. Ensuite, la détresse n'est qu'un aspect parmi tant d'autres que peut influencer la CSCS. Les prochaines études devront donc inclure d'autres variables. Autre point : seules les réponses des sous-échelles A et $\mathrm{C}$ des questionnaires PSSCAN et PSSCANR ont été retenues, et celles de la question 6 de la partie A ont été supprimées, ce qui a compliqué l'extraction des données au moment de l'examen rétrospectif des dossiers. La modification de format du questionnaire PSSCAN, survenue pendant l'étude, a également posé problème. Le questionnaire PSSCANR, dans sa nouvelle version abrégée, n'a pas encore été soumis à des tests de fiabilité et de validité. De plus, malgré que la valeur psychométrique de l'outil PSSCAN ne soit plus à faire, nous ne pouvons conclure avec certitude 
que le nombre réduit d'items du questionnaire PSSCAN-R utilisé dans la présente étude possède la même fiabilité et la même validité. Enfin, l'examen rétrospectif manuel des scores PSSCAN entre janvier 2010 et décembre 2012 était délicat; en effet, les critères d'inclusion et d'exclusion restreignaient la constitution du groupe témoin, d'où la faible taille de l'échantillon.

\section{RECOMMANDATION ET IMPLICATIONS POUR LES SOINS INFIRMIERS}

La présente étude permet de souligner l'important rôle que peut jouer la CSCS dans la trajectoire du cancer en étant présente dès le premier contact avec le milieu clinique. Cette mise en lien dès la première visite et l'accompagnement apporté au patient pour qu'il se familiarise avec le processus et les procédures sont réellement aidants. Cependant, il faudrait sans doute explorer les modes d'expression de cette assistance, ainsi que les variables pertinentes, à l'aide d'approches qualitatives afin de situer l'influence de la CSCS sur les soins du cancer dans une perspective plus vaste que la seule détresse.

\section{RÉFÉRENCES}

British Columbia Cancer Agency (2017). Intelligent patient guide to breast cancer, 6th edition. Retrieved from http://www.bccancer. bc.ca/health-info/types-of-cancer/breast-cancer/information-kit

Buxton, D., Lazenby, M., Daugherty, A., Kennedy, V., Wagner, L., Fann, J.R., \& Pirl, W. (2014). Distress Screening for Oncology Patients. Oncology Issues Journal, January-February 2014, 49-52. Retrieved from www.accc-cancer.org

Campbell, C., Craig, J., Eggert, J., \& Bailey-Dorton, C. (2010). Implementing and measuring the impact of patient navigation at a Comprehensive Community Cancer Center. Oncology Nursing Forum, 37(1), 61-68.

Carlson, L.E., Groff, S.L., Maciejewski, O., \& Bultz,B.D. (2010). Screening for distress in lung and breast cancer outpatients: A randomized controlled trial. Journal of Clinical Oncology,25(933), 4884-4891.

Case, M.A.B. (2011). Oncology nurse navigator: Ensuring safe passage. Clinical Journal of Oncology Nursing, 15(1), 33-40.

Drageset, S., Lindstrom, T.C., \& Underlid, K. (2009). Coping with breast cancer: Between diagnosis and surgery. Journal of Advanced Nursing, 66(1), 149-158.

Fillion, L., Cook, S., Veillette, A., Aubin, A., De Serres, M., Fitch, M., \& Doll, R. (2012). Professional navigation framework: Elaboration and validation in a Canadian context. Oncology Nursing Forum, 39(1), E58-69.

Hatcher, L. (2003). Step-by-step basic statistics using SAS. Cary, N.C.: SAS Institute Inc.

Hewitt M, Greenfield S, Stovall E (Eds.). (2005). From cancer patient to cancer survivor: Lost in transition. Washington, DC: National Academies Press.

Hryniuk, W., Simpson, R., McGowan, A., \& Carter, P. (2014). Patient perceptions of a comprehensive cancer navigation service. Current Oncology, 21, 69-76.

Liebert, B., Parle, M., White, K., \& Rodger, K. (2001). Establishing an evidence base for the specialist breast nurse: A model for Australian breast cancer care. Australian Health Review, 24(1), 192-200.

Lim, C.C., Devi, K., \& Ang, E. (2011). Anxiety in women with breast cancer undergoing treatment: A systemic review. International Journal of Evidence-Based Healthcare, 9, 215-235.
L'administration du questionnaire PSSCAN comme mesure de l'anxiété et de la dépression dès l'arrivée des patientes à la BCCA constitue un protocole adéquat. Nous recommandons néanmoins la tenue d'une étude évaluative pour vérifier si les réponses au questionnaire PSSCAN changent après la consultation en oncologie, ainsi que pour établir la fiabilité et la validité du questionnaire révisé (PSSCANR) malgré le nombre plus restreint d'items.

De l'avis des femmes, il était bénéfique pour elles de recevoir un appel téléphonique avant la consultation initiale en oncologie et d'être suivies après la première visite clinique (Trevillion, Singh-Carlson, Wong et Sherriff, 2015). Elles nouaient ainsi des liens avec un professionnel de la santé du centre de traitement du cancer et, du même souffle, réduisaient en partie leur anxiété devant l'inconnu. En étant attentive aux besoins d'information de cette population très diverse, la CSCS est un maillon essentiel de l'équipe de collaboration interdisciplinaire. La stratégie d'intervention de la CSCS, qui vise à soutenir les patientes dès la première consultation en oncologie, peut être le point de départ de l'établissement d'une relation patiente-infirmière fondée sur la confiance et la bienveillance, en plus d'être un bon exemple d'approche axée sur la patiente.

Linden, W., Yi, D., Barroetavena, M.C., MacKenzie, R., \& Doll, R. (2005). Development and validation of a psychosocial screening instrument for cancer. Health and Quality of Life Outcomes, 3, 54.

Linden, W., Vodermaier, A.A., MacKenzie, R., Barroetavena, M.C., Dahyum, Y., \& Doll, R. (2009). The psychosocial screen for cancer (PSSCAN): Further validation and normative data. Health and Quality of Life Outcomes, 7(16), 1-8.

National Cancer Institute. (2017). Follow-up care after cancer treatment. Retrieved from http://www.cancer.gov/about-cancer/coping/ survivorship/follow-up-care/follow-up-fact-sheet

National Coalition of Oncology Nursing Navigators. (2017). Who is the nurse navigator? Retrieved from http://www.nconn.org/

Pederson, A.E., Jo-Ann Sawatzky, J.A., Hack, T.F. (2010). The sequelae of anxiety in breast cancer: A human response to illness model. Oncology Nursing Forum, 37(4), 469-475.

Pedersen, A.E., \& Hack, T.F. (2011). The British Columbia Patient Navigation Model: A critical analysis. Oncology Nursing Forum, 38(4), 200-206.

Robinson-White, S., Conroy, B., Slavish, K.H., \& Rosenzweig, M. (2010). Patient navigation in breast cancer. Cancer Nursing, 33(2), 127-140.

Singh-Carlson, S., \& Gotz, C. (2014). Breast cancer survivorshipoptimizing follow-up care: Patients' perspectives of their practical needs. Journal of Women's Health, Issues \& Care, 3(1). Retrieved from http://www.scitechnol.com/breast-cancer-survivorshipoptimizing-followup-care-patients-perspectives-practical-needsqXS0.pdf?article_id=1750

Trevillion, K., Singh-Carlson, S., Wong, F., \& Sherriff, C. (2015). An evaluation of the nurse navigator services for the breast cancer support program. Canadian Oncology Nursing Journal, 25(4), 409-414.

Trussler, T. (2001). Uncovering the gaps: An inquiry of breast care in British Columbia. Canadian Breast Cancer Foundation BC/Yukon Chapter.

Winstead, E.R. (2012). As patient navigation expands and evolves, nurses assume many roles. National Cancer Institute, 9(15).

Zabora, J., Brintzenhofeszoc, K.B., Curbow, C., Hooker, C., \& Piantadosi, S. (2001). The prevalence of psychological distress by cancer site. Psycho-Oncology 10, 19-28. 